ANNALES

POLONICI MATHEMATICI

$80(2003)$

\title{
On perturbations of pluriregular sets generated by sequences of polynomial maps
}

\author{
by MACIEJ KlimeK (Uppsala)
}

\begin{abstract}
It is shown that an infinite sequence of polynomial mappings of several complex variables, with suitable growth restrictions, determines a filled-in Julia set which is pluriregular. Such sets depend continuously and analytically on the generating sequences, in the sense of pluripotential theory and the theory of set-valued analytic functions, respectively.
\end{abstract}

1. Introduction. Let $\mathcal{R}$ denote the set of all polynomially convex compact pluriregular subsets of $\mathbb{C}^{N}$. For any $E, F \in \mathcal{R}$ we define the distance $\Gamma(E, F)$ between $E$ and $F$ as the $L^{\infty}(E \cup F)$-distance between the pluricomplex Green functions $V_{E}$ and $V_{F}$ of $E$ and $F$ respectively. It was shown in [14] that $(\mathcal{R}, \Gamma)$ is a complete metric space. Compactness in the space $\mathcal{R}$ was investigated by Siciak in [25].

One of the most useful properties of $\Gamma$ is that it turns regular polynomial mappings into contractions of $\mathcal{R}$. More precisely, let $P: \mathbb{C}^{N} \rightarrow \mathbb{C}^{N}$ be a polynomial mapping of degree $d \geq 2$ such that $\widehat{P}^{-1}(0)=\{0\}$, where $\widehat{P}$ denotes the homogeneous part of $P$ of degree $d$. Then the set-mapping $E \mapsto P^{-1}(E)$ is a contraction. By Banach's Contraction Principle, it has a unique fixed point, which turns out to be the filled-in Julia set of $P$. This, as well as similarities between $\Gamma$ and the Hausdorff distance, have led to a number of applications in complex dynamics and yielded new examples of multivalued analytic functions (see [14]-[18]).

Analytic functions whose values are compact subsets of $\mathbb{C}$ were introduced by Oka in 1934 [20]. With the notable exception of Nishino's paper [19] and two papers by Yamaguchi ([34], [35]) the subject was dormant for nearly five decades. In 1981 Słodkowski [26] proposed a different equivalent definition of such functions, which allowed natural multidimensional gener-

2000 Mathematics Subject Classification: Primary 32U35, 32H50; Secondary 32U05, $46 \mathrm{G} 20$.

Key words and phrases: polynomial map, filled-in Julia set, pluriregular set, analytic multifunction. 
alization of the concept. In [27] he introduced yet another more restrictive multidimensional extension of Oka's definition.

It can be shown that if $A$ is a Banach algebra and $f$ is a holomorphic function with values in $A^{N}$, then the mapping $z \mapsto \sigma(f(z))$ is an analytic set-valued function, where $\sigma$ denotes the appropriate type of spectrum (see [26] if $N=1$ and [11], [30] if $N \geq 1$ ). The converse holds locally if $N=1$ (see [26]), but is only partially known in higher dimensions (see [29], [30]).

It is of great interest to construct and investigate classes of set-valued analytic functions whose values are specific types of subsets of $\mathbb{C}^{N}$. The so-called Scarcity Theorem (see [3], [4] for $N=1$ and [11] for $N \geq 1$ ) essentially tells us that analytic multifunctions whose values are finite sets are "inverses" of proper holomorphic projections of analytic varieties. Moreover, if a function has infinite sets as some values, then this must be so outside of a pluripolar set. If $z \mapsto K(z)$ is an analytic set-valued function we can also describe the size of its values in terms of being or not being pluripolar. It turns out that if the domain of definition of $K$ is connected, then either all $K(z)$ are pluripolar, or there exists a pluripolar set $F$ such that $K(z)$ is not pluripolar for $z \notin F$ (see [34] if $N=1$ and [11] if $N \geq 1$ ). One can also look at much more specific types of sets $K(z)$. Multifunctions whose values are line segments or convex polygons were investigated by Baribeau in [5] and $[6]$.

One reason why analytic set-valued functions with values in a very particular family of sets are of interest can be explained as follows. Let $\mathcal{C}$ denote a class of compact subsets of $\mathbb{C}^{N}$ with some specific properties and let $E \in \mathcal{C}$. Suppose now that $z \mapsto K(z)$ is an analytic multifunction whose values are subsets of $\mathbb{C}^{N}$. If $E=K\left(z_{0}\right)$ for some $z_{0}$, then for $z$ close to $z_{0}$ the set $K(z)$ can be viewed as an analytic perturbation of $E$. It is natural to ask if the function $K$ perturbs $E$ in such a way that the deformed version of $E$ remains within the class $\mathcal{C}$. Alternatively, we may want to characterize all or at least some multifunctions $K$ with this property. A canonical approach is to look closely at the way the set $E$ is defined. If the construction of $E$ involves some quantities that could be regarded as parameters, we can try to see if the dependence of $E$ on these parameters defines an analytic set-valued function.

Let $P=\left(P_{n}\right)_{n \geq 1}$ be a sequence of polynomial mappings such that each $P_{n}: \mathbb{C}^{N} \rightarrow \mathbb{C}^{N}$. For every point $z_{0} \in \mathbb{C}^{N}$ we define its $P$-orbit as the sequence $z_{n}=P_{n}\left(z_{n-1}\right)$ for $n \geq 1$. We also define $K_{+}[P]$ as the set of all points in $\mathbb{C}^{N}$ whose $P$-orbits are bounded. By analogy to the case when $N=1$ and $P_{1}=P_{2}=\ldots$, we will call $K_{+}[P]$ the filled-in Julia set of $P$.

The goal of this paper is to establish natural conditions under which the mapping $K_{+}: P \rightarrow K_{+}[P]$ has values in $\mathcal{R}$, is well defined on an open subset of an infinite-dimensional Banach space, is continuous and is analytic in the 
sense of the theory of analytic set-valued functions. Theorem 1 contains the main result. Examples of applications of Theorem 1 are given in the form of Theorems 2 and 3 . The former is just a strengthened version of Theorem 2 from [16], whereas the latter provides a natural construction of an analytic set-valued function whose values fail to satisfy Markov's inequality. Recall that the first example of a pluriregular set without Markov's property was discovered by Pleśniak in [21].

The paper is organized as follows. In the next two sections we describe the domain of definition of $K_{+}$and review some basic definitions concerning analytic set-valued functions. This is followed by the statement and proof of the main result. In the closing section we describe several applications of the main theorem and its relationship to other results in the literature.

2. A Banach space of sequences of polynomials. Let $d \geq 2$ be an integer. We denote by $\mathcal{P}_{d}$ the space of all polynomial mappings $P: \mathbb{C}^{N} \rightarrow$ $\mathbb{C}^{N}$ such that $\operatorname{deg}(P) \leq d$. We will view $\mathcal{P}_{d}$ as a Banach space with the norm

$$
\|P\|=\sum_{n=0}^{d}\left\|H_{n}\right\|,
$$

where $P=H_{0}+H_{1}+\ldots+H_{d}, H_{n}$ is homogeneous of degree $n$ and

$$
\left\|H_{n}\right\|=\sup _{\|z\|=1}\left\|H_{n}(z)\right\| \text {. }
$$

If $N=1$, then $\mathcal{P}_{d}$ is simply $\mathbb{C}^{d+1}$ furnished with the $\ell^{1}$ norm. Given $P \in \mathcal{P}_{d}$ it will be convenient to have the following symbols:

$$
\widehat{P}=H_{d}, \quad \widetilde{P}=H_{0}+\ldots+H_{d-1}, \quad\lfloor P\rfloor=\inf _{\|z\|=1}\|\widehat{P}(z)\| .
$$

We call $\lfloor P\rfloor$ the floor of $P$. Note that

$$
|\lfloor P\rfloor-\lfloor Q\rfloor| \leq\|P-Q\| .
$$

We say that $P$ is regular if $\widehat{P}^{-1}(0)=\{0\}$. The subset of all regular mappings in $\mathcal{P}_{d}$ is denoted by $\mathcal{P}_{d}^{*}$. It is easy to see that all regular mappings are proper.

Throughout the paper $B_{R}$ denotes the closed Euclidean ball in $\mathbb{C}^{N}$ with center at the origin and radius $R>0$. We say that $R>0$ is an escape radius for $P$ if for each $z \in \mathbb{C}^{N} \backslash B_{R}$,

$$
\lim _{n \rightarrow \infty}\|(\underbrace{P \circ \ldots \circ P}_{n \text { times }})(z)\|=\infty .
$$

For a regular mapping $P$, the formula

$$
r(P)=\frac{1+\lfloor P\rfloor+\|\widetilde{P}\|}{\lfloor P\rfloor}
$$


gives an escape radius that depends continuously on $P$ (see [16]).

Since $\operatorname{dim} \mathcal{P}_{d}<\infty$, for every $R>0$ there exists $M_{R}>0$ such that

$$
\|P\|_{B_{R}}=\sup _{z \in B_{R}}\|P(z)\| \leq M_{R}\|P\|, \quad P \in \mathcal{P}_{d} .
$$

If $E$ is a complex Banach space and $\Lambda=\left(\lambda_{j}\right)_{j \geq 1}$ is a sequence of positive numbers, then

$$
\ell_{\Lambda}^{\infty}(E)=\left\{x=\left(\xi_{n}\right)_{n \in \mathbb{N}}: \xi_{n} \in E \text { and } \sup _{n \in \mathbb{N}} \frac{\left\|\xi_{n}\right\|}{\lambda_{n}}<\infty\right\}
$$

endowed with the norm

$$
\|x\|_{\Lambda}=\sup _{n \in \mathbb{N}} \frac{\left\|\xi_{n}\right\|}{\lambda_{n}}
$$

is a Banach space. It can be called the weighted $\ell^{\infty}$ space over $E$ with weights $\Lambda$.

We will be primarily interested in the space $\ell_{\Lambda}^{\infty}\left(\mathcal{P}_{d}\right)$. For $P=\left(P_{n}\right) \in$ $\ell_{\Lambda}^{\infty}\left(\mathcal{P}_{d}\right)$ define the floor of $P$ :

We also define

$$
\lfloor P\rfloor_{\Lambda}=\inf _{n \in \mathbb{N}} \frac{\left\lfloor P_{n}\right\rfloor}{\lambda_{n}} .
$$

$$
\Omega_{\Lambda}=\left\{P \in \ell_{\Lambda}^{\infty}\left(\mathcal{P}_{d}\right):\lfloor P\rfloor_{\Lambda}>0\right\} .
$$

Since $P \mapsto\lfloor P\rfloor_{\Lambda}$ is continuous (see (1)), the set $\Omega_{\Lambda}$ is open. Another useful property of the set $\Omega_{\Lambda}$ is that the function

$$
P \mapsto \sup _{n \geq 1} r\left(P_{n}\right), \quad P=\left(P_{n}\right) \in \Omega_{\Lambda},
$$

is locally bounded from above, provided that $c=\inf _{n \in \mathbb{N}} \lambda_{n}>0$. This is so because of the estimate

$$
\sup _{n \geq 1} r\left(P_{n}\right) \leq \frac{1 / c+\|P\|_{\Lambda}}{\lfloor P\rfloor_{\Lambda}}
$$

and continuity of the right-hand side.

3. Analytic set-valued functions. Let $\operatorname{Comp}\left(\mathbb{C}^{N}\right)$ denote the family of all compact subsets of $\mathbb{C}^{N}$ and let $\Omega$ be an open subset of a Banach space $E$. If $K: \Omega \rightarrow \operatorname{Comp}\left(\mathbb{C}^{N}\right)$ is a function, we define the graph of $K$ as the set

$$
\operatorname{Graph}(K)=\left\{(z, w) \in \Omega \times \mathbb{C}^{N}: w \in K(z)\right\} .
$$

We say that the function $K$ is upper semicontinuous if for every $z_{0} \in \Omega$ and every open set $V$ such that $K\left(z_{0}\right) \subset V \subset \mathbb{C}^{N}$, there exists a neighborhood $U \subset \Omega$ of $z_{0}$ such that $K(z) \subset V$ for all $z \in U$. Equivalently, $K$ is upper semicontinuous if and only if the graph of $K$ is closed in $\Omega \times \mathbb{C}^{N}$ and the natural projection $\pi: \operatorname{Graph}(K) \rightarrow \Omega$ is proper. If $\Omega$ is locally compact, instead of assuming that $\pi$ is proper, it suffices to assume that each $a \in \Omega$ 
has a neighborhood $U$ such that $\pi^{-1}(U)$ is relatively compact in the graph of $K$ (see e.g. [9]).

Another concept we need is that of a $q$-plurisubharmonic function. For smooth functions the definition was already given in the early 1960's by Andreotti and Grauert (see [1], [2]), but its modern more general version is due to Hunt and Murray and appeared much later in 1978 (see [10]).

Let $D \subset \mathbb{C}^{n}$ be an open set. For any $q \in\{0,1, \ldots, n-1\}$ an upper semicontinuous function $u: D \rightarrow[-\infty, \infty)$ is said to be $q$-plurisubharmonic if it is subplurisuperharmonic on the intersection of $D$ with every $(q+1)$ dimensional affine subspace of $\mathbb{C}^{n}$. It can be shown that the word "subplurisuperharmonic" can be replaced by "subpluriharmonic" (see Lemma 4.4 in [31]). Obviously 0-plurisubharmonic functions are the same as plurisubharmonic functions.

Here are some fundamental properties of $q$-plurisubharmonic functions.

Properties. 1. A $\mathcal{C}^{2}$ function is q-plurisubharmonic if and only if at each point its complex Hessian has at least $(n-q)$ non-negative eigenvalues (see $[10])$.

2. If $F \subset \mathbb{C}^{n}$ is compact and $u: F \rightarrow[-\infty, \infty)$ is upper semicontinuous and q-plurisubharmonic in the interior of $F$, then $\sup u(F)=\sup u(\partial F)($ see $[10])$.

3. Being q-plurisubharmonic is a local property, invariant with respect to biholomorphic changes of coordinates.

4. The maximum of two q-plurisubharmonic functions is q-plurisubharmonic.

5. The limit of a decreasing sequence of q-plurisubharmonic functions is q-plurisubharmonic.

6. If $u$ and $v$ are $q$ - and $r$-plurisubharmonic respectively, then $u+v$ is $(q+r)$-plurisubharmonic and $\min (u, v)$ is $(q+r+1)$-plurisubharmonic (see [28], [31]).

7. Every q-plurisubharmonic function can be approximated on any compact subset $F$ of its domain by a decreasing sequence of continuous q-plurisubharmonic functions defined on a neighborhood of $F$ (see [28], [8]).

8. The upper semicontinuous regularization of the upper envelope of a family of q-plurisubharmonic functions is q-plurisubharmonic provided it is locally bounded from above (see [28], [33]).

9. A closed locally complete pluripolar set is a removable singularity for q-plurisubharmonic functions which are locally bounded from above (see [33]).

There are several ways in which analytic set-valued functions can be defined. Assume that $K: \Omega \rightarrow \operatorname{Comp}\left(\mathbb{C}^{N}\right)$ is an upper semicontinuous function. We are going to state three versions of the definition of strong 
analyticity (which are equivalent in the overlapping cases) and one definition of weak analyticity.

- If $N=1$, we say that $K$ is strongly analytic if the open set $(\Omega \times \mathbb{C}) \backslash$ $\operatorname{Graph}(K)$ is pseudoconvex (see [20] and [24]).

- If $N \geq 1$, we say that $K$ is strongly analytic if for any $(N+1)$ dimensional complex affine subspace $L$ of $E \times \mathbb{C}^{N}$, the set $Y=L \cap \operatorname{Graph}(K)$ has the local maximum property:

there is no holomorphic function $f: U \rightarrow \mathbb{C}$, where $U \subset E \times \mathbb{C}^{N}$, such that $\mid f \|_{Y}$ has a strict local maximum at a point in $U \cap Y$.

- If $\operatorname{dim} E=q$ and $N \geq 1$, we say that $K$ is strongly analytic if for every open set $\omega \subset \Omega$ and every smooth function $u$ which is $(q-1)$ plurisubharmonic in a neighborhood of $\operatorname{Graph}\left(\left.K\right|_{\omega}\right)$, the function

$$
v(z)=\sup u(\{z\} \times K(z)), \quad z \in \omega,
$$

is $(q-1)$-plurisubharmonic. (This definition and the previous one were given in $[27]$.

- If $E$ is arbitrary and $N \geq 1$, we say that $K$ is weakly analytic if for every open set $\omega \subset \Omega$ and every function $u$ plurisubharmonic in a neighborhood of $\operatorname{Graph}\left(\left.K\right|_{\omega}\right)$, the function

$$
v(z)=\sup u(\{z\} \times K(z)), \quad z \in \omega,
$$

is plurisubharmonic (see [26]).

The adverb weakly was added in [27]. In this article we need to deal with both concepts of analyticity and hence the adverb strongly has been added here to facilitate our discussion. In most of the literature only one type of multifunctions is studied at a time and thus the term analytic is sufficient.

If $E=\mathbb{C}$ and $N \geq 1$, the concepts of strong and weak analyticity are identical (see [29] and [26]). If $\operatorname{dim} E>1$, then strong analyticity implies weak analyticity, but the converse is not true as shown in the examples below. In the finite-dimensional case several equivalent definitions of strong analyticity can be found in the literature (see [27], [29], [30], [32]).

Example 1. Define

$$
\mathcal{D}:[0, \infty) \rightarrow \operatorname{Comp}(\mathbb{C}), \quad \mathcal{D}(r)=\{\zeta \in \mathbb{C}:|\zeta| \leq r\} .
$$

If $u: \Omega \rightarrow[-\infty, \infty)$ is a function, then $\mathcal{D} \circ e^{u}$ is weakly analytic if and only if $u$ is plurisubharmonic. This is one of the standard examples of weakly analytic set-valued functions exemplifying the fact that such functions generalize plurisubharmonic functions.

EXAMPLE 2. Another standard example of a weakly analytic function goes back to Yamaguchi [34] (see also [22]). We say that $K$ has local holomorphic selections if for any $z_{0} \in \Omega$ and any $w_{0} \in \partial K\left(z_{0}\right)$ there exists a 
neighborhood $U$ of $z_{0}$ and a holomorphic function $f: U \rightarrow \mathbb{C}^{N}$ such that

$$
f\left(z_{0}\right)=w_{0} \quad \text { and } \quad f(z) \in K(z) \text { for all } z \in U .
$$

If $K$ has local holomorphic selections and is upper semicontinuous, then it is weakly analytic.

In [29] Słodkowski gave a very simple example of a weakly analytic function which is not strongly analytic:

$$
S:\left(z_{1}, z_{2}\right) \mapsto \begin{cases}\{\zeta \in \mathbb{C}:|\zeta|=1\} & \text { if }\left(z_{1}, z_{2}\right) \in \mathbb{C}^{2} \backslash\{(0,0)\}, \\ \{\zeta \in \mathbb{C}:|\zeta| \leq 1\} & \text { if }\left(z_{1}, z_{2}\right)=(0,0) .\end{cases}
$$

Note that despite this, the function has local holomorphic selections.

The choice between weak and strong analyticity is sometimes dictated by the context. If some kind of analytic structure of the graph of a multifunction is of primary interest, strong analyticity seems to be the right choice. The key reason was provided in [32], where it was shown that any strongly analytic function is a decreasing limit of locally trivial strongly analytic functions, that is, functions which are locally unions of graphs of families of holomorphic functions. On the other hand, if properties related to plurisubharmonic functions are in focus - as in this paper - weak analyticity seems to be somewhat more appropriate. An excellent exposition of the theory of weakly analytic multifunctions is given in [22] (see also [23]).

4. The main result. In the statement of the main result we use the notation from the introduction.

THEOREM 1. Let $\Lambda=\left(\lambda_{j}\right)_{j \geq 1}$ be a sequence of numbers such that $\inf _{n \in \mathbb{N}} \lambda_{n}>0$ and

$$
\sum_{n=1}^{\infty} \frac{\lambda_{n}}{d^{n}}<\infty
$$

Then the set-valued mapping

$$
K_{+}: P \mapsto K_{+}[P], \quad \Omega_{\Lambda} \rightarrow \mathcal{R},
$$

is well defined, continuous and weakly analytic.

Proof. According to (3) for each point in $\Omega_{\Lambda}$ we can choose a neighborhood $\omega$ and a number $R>1$ such that if $P=\left(P_{n}\right) \in \omega$, then $R$ is an escape radius common to all $P_{n}$ 's. Since the conclusion of the theorem is local we can work with such a neighborhood $\omega$ instead of $\Omega_{\Lambda}$. Moreover, we may also suppose that there exists a constant $L>1$ such that $\left\|P_{n}(z)\right\| \geq L\|z\|$ for all $P=\left(P_{n}\right) \in \omega$ and $z \in \mathbb{C}^{N} \backslash B_{R}$. In what follows, $M_{R}$ is defined as in (2).

For $P=\left(P_{n}\right) \in \omega$ define

$$
E_{n}^{P}=\left(P_{n} \circ P_{n-1} \circ \ldots \circ P_{1}\right)^{-1}\left(B_{R}\right), \quad n \in \mathbb{N} \text {. }
$$


Note that $E_{n+1}^{P} \subset E_{n}^{P}$, because $P_{n+1}^{-1}\left(B_{R}\right) \subset B_{R}$. Furthermore

$$
K_{+}[P]=\bigcap_{n \in \mathbb{N}} E_{n}^{P} .
$$

Hence in order to show that $K_{+}[P] \in \mathcal{R}$, it suffices to check that the sequence $\left(E_{n}^{P}\right)$ is convergent in $\mathcal{R}$ (see e.g. Corollary 5.1.2 in [13]). In view of Theorem 5.3 .1 in [13],

$$
\begin{aligned}
\Gamma\left(E_{n+1}^{P}, E_{n}^{P}\right) & \leq \frac{\Gamma\left(P_{n+1}^{-1}\left(B_{R}\right), B_{R}\right)}{d^{n}} \\
& =\frac{\log ^{+}\left(\left\|P_{n+1}\right\|_{B_{R}} / R\right)}{d^{n+1}} \\
& \leq \frac{M_{R}\|P\|_{\Lambda} \lambda_{n+1}}{R d^{n+1}}
\end{aligned}
$$

and so $\left(E_{n}^{P}\right)$ is a Cauchy sequence because of (4) and the triangle inequality.

To show continuity of $K_{+}$we proceed as follows. First, if $P, Q \in \omega$, the estimate in (5) implies that

$$
\begin{aligned}
\Gamma\left(E_{n+1}^{P}, E_{n+1}^{Q}\right) & =\Gamma\left(E_{n+1}^{P}, E_{n}^{P}\right)+\Gamma\left(E_{n}^{P}, E_{n}^{Q}\right)+\Gamma\left(E_{n}^{Q}, E_{n+1}^{Q}\right) \\
& \leq 2 \frac{M_{R} \max \left\{\|P\|_{\Lambda},\|Q\|_{\Lambda}\right\} \lambda_{n+1}}{R d^{n+1}}+\Gamma\left(E_{n}^{P}, E_{n}^{Q}\right)
\end{aligned}
$$

for all $n$. Consequently,

$$
\begin{aligned}
\Gamma\left(E_{n+m}^{P}, E_{n+m}^{Q}\right) \leq & 2 \frac{M_{R} \max \left\{\|P\|_{\Lambda},\|Q\|_{\Lambda}\right\}}{R} \sum_{k=n+1}^{n+m} \frac{\lambda_{k}}{d^{k}} \\
& +\Gamma\left(E_{n}^{P}, E_{n}^{Q}\right) .
\end{aligned}
$$

Take $\varepsilon>0$ and fix $P \in \omega$. Choose $r>0$ so that if $\|P-Q\|_{\Lambda}<r$, then $Q \in \omega$. By choosing a sufficiently large $n$ and letting $m \rightarrow \infty$ in (6) we get (because of (4))

$$
\Gamma\left(K_{+}[P], K_{+}[Q]\right) \leq \frac{\varepsilon}{2}+\Gamma\left(E_{n}^{P}, E_{n}^{Q}\right)
$$

But

$$
\begin{aligned}
\Gamma\left(E_{n}^{P}, E_{n}^{Q}\right) & =\frac{1}{d^{n}} \sup _{z \in B_{R}}\left|\log ^{+} \frac{\left\|\left(P_{n} \circ \ldots \circ P_{1}\right)(z)\right\|}{R}-\log ^{+} \frac{\left\|\left(Q_{n} \circ \ldots \circ Q_{1}\right)(z)\right\|}{R}\right| \\
& \leq \frac{\left\|\left(P_{n} \circ \ldots \circ P_{1}\right)-\left(Q_{n} \circ \ldots \circ Q_{1}\right)\right\|_{B_{R}}}{d^{n} R}
\end{aligned}
$$

and the right-hand side can be made smaller than $\varepsilon / 2$ if $Q$ is sufficiently close to $P$.

If $E \in \mathcal{R}$, then the sets $\left\{z \in \mathbb{C}^{N}: V_{E}(z)<\varepsilon\right\}$, where $\varepsilon>0$, form a base of neighborhoods of $E$ (see e.g. [14]). Hence, if $(X, d)$ is a metric space and a mapping $F:(X, d) \rightarrow(\mathcal{R}, \Gamma)$ is continuous, then $F$ is also upper 
semicontinuous. In particular, we have shown that $K_{+}: \Omega_{\Lambda} \rightarrow \mathcal{R}$ is upper semicontinuous.

The next objective is to show that $K_{+}$is actually analytic. To this end we will prove four statements which are also interesting in their own right.

Remark 1. Let $r>0$. The mapping $P \mapsto P^{-1}\left(B_{r}\right)$, from $\mathcal{P}_{d}^{*}$ to $\mathcal{R}$, is continuous. In particular, it is upper semicontinuous.

As above, this is so because

$$
\left|V_{P^{-1}\left(B_{r}\right)}(z)-V_{Q^{-1}\left(B_{r}\right)}(z)\right| \leq \frac{1}{d r}\|P(z)-Q(z)\|, \quad z \in \mathbb{C}^{N} .
$$

Remark 2 (see also [16]). Let $a \in \mathbb{C}^{N}$. The set-valued mapping $P \mapsto$ $P^{-1}(a)$ defined on $\mathcal{P}_{d}^{*}$ is strongly analytic.

Without loss of generality we may suppose that $a=0$. It is easy to check that the set

$$
A=\left\{(P, z) \in \mathcal{P}_{d}^{*} \times \mathbb{C}^{N}: P(z)=0\right\}
$$

is a closed analytic submanifold of $\mathcal{P}_{d}^{*} \times \mathbb{C}^{N}$ of dimension $\operatorname{dim} \mathcal{P}_{d}$. Let $\pi$ : $A \rightarrow \mathcal{P}_{d}^{*}$ denote the natural projection $(P, z) \mapsto P$. Given $P_{0} \in \mathcal{P}_{d}^{*}$, let $r>0$ be chosen so that if $Q \in K_{P_{0}, r}=\left\{P \in \mathcal{P}_{d}^{*}:\left\|P-P_{0}\right\| \leq r\right\}$, then $r(Q) \leq r\left(P_{0}\right)+1$. Then $\pi^{-1}\left(K_{P_{0}, r}\right) \subset K_{P_{0}, r} \times B_{r\left(P_{0}\right)+1}$. This implies that the natural projection $\pi$ is proper. Because of this, for any neighborhood $U$ of $P^{-1}(0)$ we can find $s>0$ such that $P^{-1}\left(B_{s}\right) \subset U$. Hence, in view of Remark 1, $P \mapsto P^{-1}(0)$ is upper semicontinuous. Finally, since $\pi: A \rightarrow$ $\mathcal{P}_{d}^{*}$ is a branched covering, we get strong analyticity, because the relevant properties of $q$-plurisubharmonic functions are virtually the same as in the plurisubharmonic case.

Remark 3 (see also [16]). Let $r>0$. The mapping $P \mapsto P^{-1}\left(B_{r}\right)$, from $\mathcal{P}_{d}^{*}$ to $\mathcal{R}$, is strongly analytic.

We already have upper semicontinuity. Let $q=\operatorname{dim} \mathcal{P}_{d}$. If $u$ is $(q-1)$ plurisubharmonic in a neighborhood $V$ of $\{P\} \times P^{-1}\left(B_{r}\right)$, the conclusion follows directly from Remark 2 and the equality

$$
\sup u\left(\{Q\} \times Q^{-1}\left(B_{r}\right)\right)=\sup _{a \in B_{r}} \sup u\left(\{Q\} \times Q^{-1}(a)\right), \quad Q \in V .
$$

REMARK 4. Let $\Omega$ be an open subset in a complex Banach space $E$. Suppose that $K_{n}: \Omega \rightarrow \mathcal{R}$, for $n \geq 1$, is a sequence of weakly analytic multifunctions which is pointwise convergent to an upper semicontinuous set-valued function $K: \Omega \rightarrow \mathcal{R}$. Then $K$ is also weakly analytic.

Weak analyticity of an upper semicontinuous set-valued function is equivalent to weak analyticity on complex affine lines. Hence in view of Theorem 1 in [12], in order to prove the above statement it is enough to check that for any function $u \in \operatorname{PSH}\left(E \times \mathbb{C}^{N}\right)$ with logarithmic growth at 
infinity the function $z \mapsto \sup u(\{z\} \times K(z))$ is plurisubharmonic in $\Omega$. But this is true because this function is upper semicontinuous and for $z \in \Omega$ we have the estimate

$$
\left|\sup u\left(\{z\} \times K_{n}(z)\right)-\sup u(\{z\} \times K(z))\right| \leq \Gamma\left(K_{n}(z), K(z)\right) .
$$

We complete the proof of the theorem by applying Remarks 3 and 4 to the set-valued functions $P \mapsto E_{n}^{P}$.

5. Applications. Special cases of the analyticity part of Theorem 1 can be found in the literature. In the case of $N=1$, weak analyticity of the mapping $P \mapsto K_{+}[(P, P, \ldots)]$ which is defined on the set of all polynomials of degree $d$ of one complex variable follows from a result of Baribeau and Ransford [7] combined with the upper semicontinuity property shown by Douady [9]. In this instance $K_{+}[(P, P, \ldots)]$ is simply the filled-in Julia set associated with the polynomial $P$. Douady's paper also contains a discussion of discontinuity of this mapping if the distance between two compact sets in $\mathbb{C}$ is measured in the sense of the Hausdorff metric.

For $N \geq 1$, weakly analytic dependence of the composite Julia set generated by a finite family of polynomials on these polynomials was shown in [16]. Let

$$
\Sigma_{k}=\{1, \ldots, k\}^{\mathbb{N}} .
$$

It is convenient to define the auxiliary function

$$
\mathbf{s e q}_{\sigma}:\left(\mathcal{P}_{d}^{*}\right)^{k} \rightarrow\left(\mathcal{P}_{d}^{*}\right)^{\mathbb{N}}
$$

by the formula

$$
\operatorname{seq}_{\sigma}\left(P_{1}, \ldots, P_{k}\right)=\left(P_{\sigma(n)}\right)_{n \in \mathbb{N}}
$$

where $\sigma \in \Sigma_{k}$.

If $d \geq 2$, then the composite Julia set generated by the polynomials $P_{1}, \ldots, P_{k} \in \mathcal{P}_{d}^{*}$ is defined to be

$$
K_{+}^{\mathrm{c}}\left[P_{1}, \ldots, P_{k}\right]=\left(\bigcup_{\sigma \in \Sigma_{k}} K_{+}\left[\mathbf{s e q}_{\sigma}\left(P_{1}, \ldots, P_{k}\right)\right]\right)^{\wedge} \in \mathcal{R},
$$

where the "hat" denotes the operation of taking the polynomially convex hull of the expression in parentheses (see [15]).

Theorem 2. Let $d \geq 2$. The set-valued mappings

$$
K_{+} \circ \mathbf{s e q}_{\sigma}:\left(\mathcal{P}_{d}^{*}\right)^{k} \rightarrow \mathcal{R}, \quad \sigma \in \Sigma_{k},
$$

and

$$
K_{+}^{\mathrm{c}}:\left(\mathcal{P}_{d}^{*}\right)^{k} \rightarrow \mathcal{R}
$$


are continuous. Moreover the mappings $K_{+} \circ \mathbf{o s e q}_{\sigma}$ are strongly analytic and $K_{+}^{\mathrm{c}}$ is weakly analytic.

Proof. Theorem 1 yields continuity of the functions $K_{+} \circ{ }^{\circ} \mathbf{s e q}_{\sigma}$ and hence also their upper semicontinuity.

Using the same argument as in the proof of Theorem 1 we check that given $\varepsilon>0$ and $P \in\left(\mathcal{P}_{d}^{*}\right)^{k}$, there exists a neighborhood $\omega \subset\left(\mathcal{P}_{d}^{*}\right)^{k}$ of $P$ such that

$$
\Gamma\left(K_{+}\left[\mathbf{s e q}_{\sigma}(P)\right], K_{+}\left[\mathbf{s e q}_{\sigma}(Q)\right]\right) \leq \varepsilon
$$

for all $Q \in \omega$ and all $\sigma \in \Sigma_{k}$. Thus

$$
\begin{aligned}
\Gamma\left(K_{+}^{\mathrm{c}}[P], K_{+}^{\mathrm{c}}[Q]\right) & =\Gamma\left(\bigcup_{\sigma \in \Sigma_{k}} K_{+}\left[\mathbf{s e q}_{\sigma}(P)\right], \bigcup_{\sigma \in \Sigma_{k}} K_{+}\left[\mathbf{s e q}_{\sigma}(Q)\right]\right) \\
& \leq \sup _{\sigma \in \Sigma_{k}} \Gamma\left(K_{+}\left[\mathbf{s e q}_{\sigma}(P)\right], K_{+}\left[\mathbf{s e q}_{\sigma}(Q)\right] \leq \varepsilon\right.
\end{aligned}
$$

for all $Q \in \omega$. Consequently, $K_{+}^{\mathrm{c}}$ is $\Gamma$-continuous and hence also upper semicontinuous.

Fix $\sigma \in \Sigma_{k}$. Let $q=\operatorname{dim}\left(\mathcal{P}_{d}\right)^{k}$ and let $u$ be a smooth $(q-1)$-plurisubharmonic function in a neighborhood $U \times V \subset\left(\mathcal{P}_{d}^{*}\right)^{k} \times \mathbb{C}^{N}$ of the set

$$
\left\{\left(P_{1}, \ldots, P_{k}\right)\right\} \times K_{+}\left(\mathbf{s e q}_{\sigma}\left(P_{1}, \ldots, P_{k}\right)\right) .
$$

By choosing a smaller $U$ if necessary, we can ensure that there exist $R>0$ and $L>1$ such that for each $\left(Q_{1}, \ldots, Q_{k}\right) \in U$ :

(a) $R$ is a radius of escape for $Q_{1}, \ldots, Q_{k}$;

(b) $\left\|Q_{n}(z)\right\| \geq L\|z\|$ for $n=1, \ldots, k$ and $z \in \mathbb{C}^{N} \backslash B_{R}$.

For $\left(Q_{1}, \ldots, Q_{k}\right) \in U$ we define $E_{n}^{Q}$, where $Q=\operatorname{seq}_{\sigma}\left(Q_{1}, \ldots, Q_{k}\right)$, just as in the proof of Theorem 1 . In view of Remark 3,

$$
\left(Q_{1}, \ldots, Q_{k}\right) \mapsto E_{n}^{Q}
$$

is strongly analytic in $U$, the sequence $\left(E_{n}^{Q}\right)_{n \in \mathbb{N}}$ is decreasing with respect to inclusion and

$$
K_{+}\left[\operatorname{seq}_{\sigma}\left(Q_{1}, \ldots, Q_{k}\right)\right]=\bigcap_{n \in \mathbb{N}} E_{n}^{Q} .
$$

Since

$$
\begin{aligned}
\sup u\left(\left\{\left(Q_{1}, \ldots, Q_{k}\right)\right\} \times E_{n}^{Q}\right) \\
\searrow \sup u\left(\left\{\left(Q_{1}, \ldots, Q_{k}\right)\right\} \times K_{+}\left[\operatorname{seq}_{\sigma}\left(Q_{1}, \ldots, Q_{k}\right)\right]\right)
\end{aligned}
$$

we get strong analyticity of $K_{+} \circ \mathbf{s e q}_{\sigma}$.

Weak analyticity of $K_{+}^{\mathrm{c}}$ was shown in [16].

Theorem 1 also yields a significant generalization of examples of pluriregular sets on which Markov's inequality fails, which were given in [16]. Recall 
that a compact set $E \subset \mathbb{C}^{N}$ has Markov's property if there exist positive constants $M, r$ such that

$$
\|\operatorname{grad} P\|_{E} \leq M(\operatorname{deg} P)^{r}\|P\|_{E}
$$

for every complex polynomial $P$ of $N$ complex variables.

Suppose now that $N=1$. Let $\mathcal{M}_{k}$ be the set of all complex matrices with infinitely many rows and $k$ columns. By identifying polynomials with their coefficients we can identify $\mathcal{P}_{d}$ with $\mathbb{C}^{d+1}$ (with the $\ell^{1}$ norm) and we can view $\ell_{\Lambda}^{\infty}\left(\mathbb{C}^{d+1}\right)$ as a subset of $\mathcal{M}_{d+1}$. Let $\Lambda \in \mathcal{M}_{d+1}$ have positive entries. Then $\ell_{\Lambda}^{\infty}(\mathbb{C})$ is well defined. If $\Lambda$ consists of $d+1$ identical columns and $\Lambda^{\prime}$ denotes any of them, then of course

$$
\ell_{\Lambda}^{\infty}(\mathbb{C})=\ell_{\Lambda^{\prime}}^{\infty}\left(\mathbb{C}^{d+1}\right)
$$

where $\mathbb{C}^{d+1}$ is equipped with the $\ell^{\infty}$ norm. Our earlier choice of the norm in $\mathcal{P}_{d}$ was the most natural in the case of polynomials of several variables. In the one-variable case we can just as well identify $\mathcal{P}_{d}$ with $\mathbb{C}^{d+1}$ with the $\ell^{\infty}$ norm.

Theorem 3. Let $d \geq 2$ and let $\Lambda=\left(\lambda_{j}\right)_{j \geq 1}$ be a sequence of numbers such that $\inf _{n \in \mathbb{N}} \lambda_{n}>0, \sum_{n=1}^{\infty} \lambda_{n} / d^{n}<\infty$ and

$$
\lim _{n \rightarrow \infty}\left(\lambda_{1} \cdot \ldots \cdot \lambda_{n}\right)^{1 / n}=\infty \text {. }
$$

Let

$$
\omega_{\Lambda}=\left\{\left[a_{i j}\right] \in \ell_{\Lambda}^{\infty}\left(\mathbb{C}^{d}\right): \inf _{i \in \mathbb{N}} \frac{\left|a_{i d}\right|}{\lambda_{i}}>0 \text { and } \sup _{i \in \mathbb{N}}\left|\frac{a_{i 1}}{\lambda_{i}}-1\right|<\frac{1}{2}\right\} .
$$

For $A=\left[a_{i j}\right] \in \omega_{\Lambda}$ define

$$
K_{+}[A]=K_{+}\left[\left(P_{1}, P_{2}, \ldots\right)\right],
$$

where $P_{i}(z)=a_{i 1} z+a_{i 2} z^{2}+\ldots+a_{i d} z^{d}$ for $i \geq 1$. Then $\omega_{\Lambda}$ is open in $\ell_{\Lambda}^{\infty}\left(\mathbb{C}^{d}\right)$, the multifunction $A \mapsto K_{+}[A]$ is weakly analytic and all of its values are pluriregular sets without Markov's property.

Proof. Openness of $\omega_{\Lambda}$ is obvious. It is also clear that the mapping

$$
\left(a_{i 1}, \ldots, a_{i d}\right) \mapsto\left(0, a_{i 1}, \ldots, a_{i d}\right)
$$

embeds $\omega_{\Lambda}$ into $\Omega_{\Lambda}$, and thus weak analyticity of $A \mapsto K_{+}[A]$ follows from Theorem 1 . To show that $K_{+}[A]$ does not have Markov's property for a given $A$, define

$$
Q_{n}=P_{n} \circ \ldots \circ P_{1},
$$

where $P_{i}$ are as in the statement of Theorem 3 . Note that $0 \in K_{+}[A]$ because 0 is a fixed point of all $P_{i}^{\prime}$ 's. Note also that $Q_{n}^{\prime}(0)=a_{n, 1} \cdot a_{n-1,1} \cdot \ldots \cdot a_{1,1}$. If $R>1$ is a common escape radius for all $P_{i}$ 's, then $\left|Q_{n}(z)\right| \leq R$ for all $z \in K_{+}[A]$. If Markov's inequality were satisfied, we would have

$$
\left|a_{n, 1} \cdot a_{n-1,1} \cdot \ldots \cdot a_{1,1}\right| \leq M d^{n r} R
$$


for some $M, r>0$ and for all $n \in \mathbb{N}$. But since $A \in \omega_{\Lambda}$ we have the estimate $\left|a_{i, 1}\right|>\lambda_{i} / 2$. Therefore (8) would contradict (7).

\section{References}

[1] A. Andreotti, Théorèmes de dépendance algébrique sur les espaces complexes pseudoconcaves, Bull. Soc. Math. France 91 (1963), 1-38.

[2] A. Andreotti et $\mathrm{H}$. Grauert, Théorèmes de finitude pour la cohomologie des espaces complexes, ibid. 90 (1962), 193-259.

[3] B. Aupetit, Propriétés spectrales des algèbres de Banach, Lecture Notes in Math. 735, Springer, 1979.

[4] - Analytic multivalued functions in Banach algebras and uniform algebras, Adv. Math. 44 (1982), 18-60.

[5] L. Baribeau, Sur les fonctions analytiques multiformes dont les valeurs sont des segments, Canad. Math. Bull. 33 (1990), 100-105.

[6] —, Multifonctions analytiques polygonales, Studia Math. 96 (1990), 167-173.

[7] L. Baribeau and T. J. Ransford, Meromorphic multifunctions in complex dynamics, Ergodic Theory Dynam. Systems 12 (1992), 39-52.

[8] L. Bungart, Piecewise smooth approximations to q-plurisubharmonic functions, Pacific J. Math. 142 (1990), 227-244.

[9] A. Douady, Does a Julia set depend continuously on the polynomial?, in: Proc. Sympos. Appl. Math. 49, Amer. Math. Soc., 1994, 91-138.

[10] L. R. Hunt and J. J. Murray, q-plurisubharmonic functions and a generalized Dirichlet problem, Michigan Math. J. 25 (1978), 299-316.

[11] M. Klimek, Joint spectra and analytic set-valued functions, Trans. Amer. Math. Soc. 294 (1986), 187-196.

[12] - , A criterion of analyticity for set-valued functions, Proc. Roy. Irish Acad. Sect. A 86 (1986), 1-4.

[13] —, Pluripotential Theory, Oxford Univ. Press, 1991.

[14] - Metrics associated with extremal plurisubharmonic functions, Proc. Amer. Math. Soc. 123 (1995), 2763-2770.

[15] - Inverse iteration systems in $\mathbb{C}^{N}$, in: Complex Analysis and Differential Equations (Proc. Marcus Wallenberg Sympos. in Honour of Matts Essén, Uppsala, 1997), Acta Univ. Upsaliensis Skr. Uppsala Univ. C Organ. Hist. 64, Uppsala Univ., 1999, 206-214.

[16] —, Iteration of analytic multifunctions, Nagoya Math J. 162 (2001), 19-40.

[17] M. Kosek, Hölder continuity property of filled-in Julia sets in $\mathbb{C}^{n}$, Proc. Amer. Math. Soc. 125 (1997), 2029-2032.

[18] —, Hölder continuity property of composite Julia sets, Bull. Polish Acad. Sci. Math. 46 (1998), 391-399.

[19] T. Nishino, Sur les ensembles pseudoconcaves, J. Math. Kyoto Univ. 1 (1962), 225245.

[20] K. Oka, Note sur les familles des fonctions analytiques multiformes etc., J. Sci. Hiroshima Univ. Ser. A 4 (1934), 93-98.

[21] W. Pleśniak, A Cantor regular set which does not have Markov's property, Ann. Polon. Math. 51 (1990), 269-274.

[22] T. J. Ransford, Analytic multivalued functions, essay presented to the Trinity College Research Fellowship Competition, Cambridge Univ., 1983. 
[23] T. J. Ransford, Open mapping, inversion and implicit function theorems for analytic multivalued functions, Proc. London Math. Soc. 49 (1984), 537-562.

[24] - Holomorphic processes in Banach spaces and Banach algebras, J. Multivariate Anal. 48 (1994), 315-346.

[25] J. Siciak, On metrics associated with extremal plurisubharmonic functions, Bull. Polish Acad. Sci. Math. 45 (1997), 151-161.

[26] Z. Słodkowski, Analytic set-valued functions and spectra, Math. Ann. 256 (1981), 363-386.

[27] —, Analytic multifunctions, q-plurisubharmonic functions and uniform algebras, in: Proc. Conf. Banach Algebras and Several Complex Variables, F. Greenleaf and D. Gulick (eds.), Contemp. Math. 32, Amer. Math. Soc., 1984, 243-258.

[28] -, The Bremermann-Dirichlet problem for q-plurisubharmonic functions, Ann. Scuola Norm. Sup. Pisa (4) 11 (1984), 303-326.

[29] -, An analytic set-valued selection and its applications to the corona theorem, to polynomial hulls and joint spectra, Trans. Amer. Math. Soc. 294 (1986), 367-377.

[30] -, Analytic perturbation of the Taylor spectrum, ibid. 297 (1986), 319-336.

[31] - Local maximum property and q-plurisubharmonic functions in uniform algebras, J. Math. Anal. Appl. 115 (1986), 105-130.

[32] - Approximation of analytic multifunctions, Proc. Amer. Math. Soc. 105 (1989), 387-396.

[33] V. Vâjâitu, A Levi problem for continuous strongly q-plurisubharmonic functions, C. R. Acad. Sci. Paris 328 (1999), 573-578.

[34] H. Yamaguchi, Sur une uniformité des surfaces constantes d'une fonction entière de deux variables complexes, J. Math. Kyoto Univ. 13 (1973), 417-433.

[35] —, Sur le mouvement des constantes de Robin, ibid. 15 (1975), 53-71.

Department of Mathematics

Uppsala University

P.O. Box 480

751-06 Uppsala, Sweden

E-mail: maciej.klimek@math.uu.se

Reçu par la Rédaction le 4.12.2001

Révisé le 27.3.2002 